\title{
BlaVar: A numerical study of long-term multi-wavelength blazar variability
}

\section{Polkas, ${ }^{a, *}$ M. Petropoulou, ${ }^{a}$ G. Vasilopoulos, ${ }^{b, c}$ A. Mastichiadis, ${ }^{a}$ C. M. Urry, ${ }^{c}$ P. Coppi $^{c}$ and C. Bailyn ${ }^{c}$}

${ }^{a}$ Department of Physics, National \& Kapodistrian University of Athens, University Campus, Zografos, 15784, Greece

${ }^{b}$ Université de Strasbourg, CNRS, Observatoire astronomique de Strasbourg, UMR 7550, 67000, Strasbourg, France

${ }^{c}$ Department of Astronomy, Yale University, PO Box 208101, New Haven, CT 06520-8101, USA

E-mail: markpolkas@phys.uoa.gr

Blazars are characterized by flux variability that is frequency-dependent and manifests in a variety of timescales. Decade-long monitoring of blazars at optical and infrared (OIR) wavelengths with the Small and Moderate Aperture Research Telescope System (SMARTS) in Chile and in gamma-rays with the Fermi Large Area Telescope (LAT) has enabled the systematic study of their multi-wavelength (MW) long-term variability. These studies pinpoint that besides extreme shortterm variability (minutes to hours), a source can exhibit correlated and uncorrelated inter-band flux variability between different observation periods and/or on different timescales (days to years). In this work, we investigate from a theoretical perspective the long-term variability properties of blazar emission. To do so, we impose variations on the main parameters of the one-zone leptonic model (injection luminosity of relativistic electrons, strength of magnetic field, Doppler factor, and external photon field luminosity) motivated by the Fermi-LAT full-mission light curves of blazars. Using as case studies two bright and well-monitored blazars from the SMARTS sample (PKS2155-304 and 3C273), we compute 10 year-long OIR, X-ray, and gamma-ray model light curves for different varying parameters. We compare the findings of our theoretical investigation with MW observations using various measures of variability. While no single-varying parameter simulation can explain all MW variability properties, our results motivate future time-dependent studies with coupling between two or more physical parameters to describe the MW long-term blazar variability.

$37^{\text {th }}$ International Cosmic Ray Conference (ICRC 2021)

July 12th - 23rd, 2021

Online - Berlin, Germany

\footnotetext{
${ }^{*}$ Presenter
} 


\section{Introduction}

Blazars are the most powerful persistent astrophysical sources of non-thermal electromagnetic radiation in the Universe, with spectral energy distributions (SEDs) spanning $\sim 15$ decades in energy, from radio frequencies up to high-energy $\gamma$-rays. Blazars are also characterized by flux variability that is frequency-dependent and manifests on a variety of timescales, ranging from minutes to years [for a review, see 5].

Understanding the origin of blazar flares has been the focus of many theoretical studies [e.g. $2,12,14,17]$. Modeling the long-term variability properties of blazar emission, however, has drawn less attention mostly because of the sparse multi-wavelength (MW) coverage of blazars on year-long timescales. The Large Area Telescope (LAT) on board the Fermi Gamma-Ray Space Telescope is a unique instrument due to its near-continuous $\gamma$-ray monitoring of blazars [3]. With an $\sim 11 \mathrm{yr}$ operation period, Fermi-LAT produced a large sample of long-term blazar $\gamma$-ray light curves with regular sampling, which enabled cross-correlation studies of $\gamma$-ray light curves with radio and/or optical light curves [e.g. 13, 22]. The meter-class telescopes of the Small and Moderate Aperture Research Telescope System (SMARTS) have produced good quality optical/infrared (OIR) light curves with regular cadence for a large sample of bright southern $\gamma$-ray blazars as part of the Yale/SMARTS blazar monitoring program ${ }^{1}$.

Motivated by the availability of decade-long data at OIR wavelengths and $\mathrm{GeV} \gamma$-rays (see Fig. 1), we investigate the long-term variability properties of blazar emission by introducing an observationally motivated time-dependence to four main parameters of the one-zone leptonic model: electron compactness ${ }^{2}\left(l_{\mathrm{e}}\right)$, strength of magnetic field $(B)$, Doppler factor $(\delta)$ and external photon field compactness $\left(l_{\mathrm{ext}}\right)$. Using the probability density function (PDF) and the power spectral density (PSD) of the observed decade-long Fermi-LAT light curves, we create synthetic $\gamma$-ray light curves and variation patterns for the model parameters in order to simulate the long-term MW flux variability. We compare our findings with observations of two bright blazars from the SMARTS sample: PKS 2155-304 [ $z=0.116,1]$, a BL Lac object which is known for its weak correlation between optical and $\mathrm{GeV} \gamma$-rays at low flux states [10], and the well-known FSRQ 3C $273[z=0.158$, 20], whose optical/UV spectrum shows a prominent excess of emission interpreted as a contribution from a luminous accretion disc [21].

\section{Modeling flux variability}

We assume that the broadband blazar emission is produced in a localized region of the jet. This is modeled as a spherical region (blob) of comoving radius $R$ with a tangled magnetic field of strength $B$. Accelerated electrons with a power-law energy distribution $\left(N_{e}(\gamma) \propto \gamma^{-p}, \gamma_{\min }<\gamma<\gamma_{\max }\right)$ are injected into this region where they subsequently lose energy via synchrotron and inverse Compton processes, while they can escape from it on a timescale equal to the light crossing time of the source. The blob is moving with a Lorentz factor $\Gamma$ with respect to an observer at angle $\theta_{\text {obs. }}$ The radiation in the observer's frame is Doppler boosted, with the Doppler factor defined as $\delta=\Gamma^{-1}\left(1-\beta \cos \left(\theta_{\mathrm{obs}}\right)\right)^{-1}$ and $\beta=\sqrt{1-1 / \Gamma^{2}}$.

\footnotetext{
${ }^{1}$ http://www .astro.yale.edu/smarts/glast/home.php

${ }^{2}$ This is defined as $l=\sigma_{T} L / 4 \pi R m_{e} c^{3}$, where $L$ is the luminosity.
} 

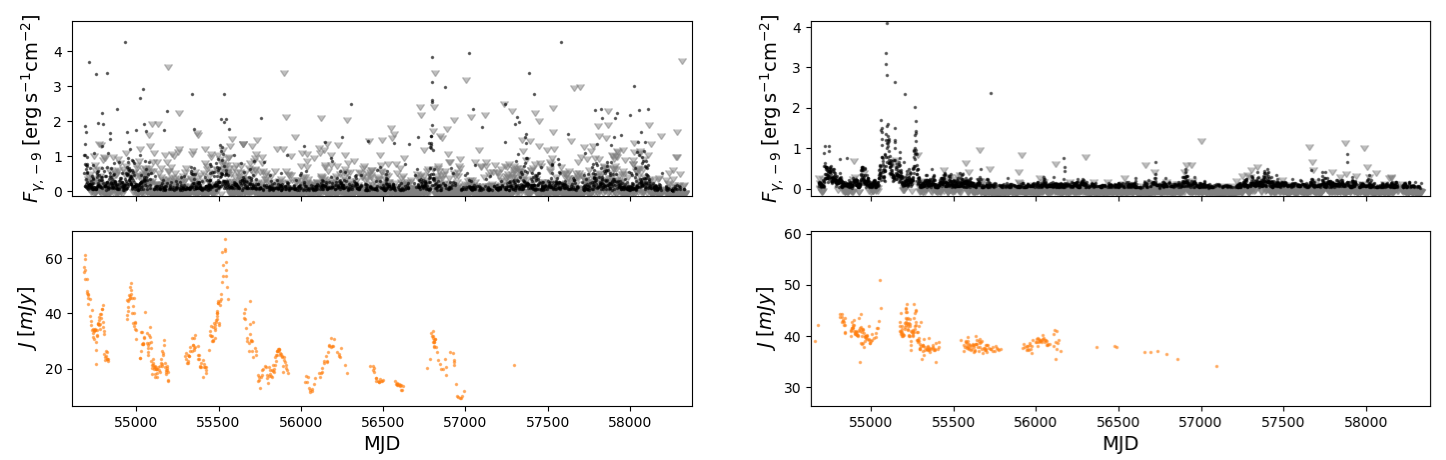

Figure 1: Daily binned Fermi-LAT light curves with grey markers indicating upper limits (top) and J-band SMARTS light curves (bottom) for PKS 2155-304 and 3C 273 (left and right panels, respectively).

When all parameters describing the emitting region and the particle distribution are constant in time, no changes in the emitted flux are expected. To model the long-term blazar flux variability we follow the procedure outlined below.

1. We create a synthetic light curve, $\tilde{F}_{\mathrm{LAT}}$, with the same timing properties as the observed Fermi-LAT light curve. For this purpose, we adopt the method of Ref. [8] that uses both the PDF and the PSD of the real LAT light curve (for details, see Ref. [16]).

2. We compute a steady-state model for the blazar SED, which sets the initial conditions for the time-dependent calculations. Using publicly available data from SSDC, we build timefiltered SEDs, each one comprised of observations taken within $2 \mathrm{yr}$ time intervals in $10 \mathrm{yr}$. The purpose of using the $2 \mathrm{yr}$ data sets is to illustrate the variability of the source on those timescales.

3. We calculate a time series for the model parameter $x(t)^{3}$. We use a transformation of the form

$$
x\left(t_{\mathrm{j}}\right)=x_{0}\left(\frac{\tilde{F}_{\mathrm{LAT}}\left(t_{\mathrm{j}}\right)}{\left\langle\tilde{F}_{\mathrm{LAT}}\left(t_{\mathrm{j}}\right)\right\rangle}\right)^{1 / \sigma_{\gamma}}
$$

where $x_{0}$ is the steady-state value of the time-dependent parameter, $\tilde{F}_{\mathrm{LAT}}\left(t_{\mathrm{j}}\right)$ and $\left\langle\tilde{F}_{\mathrm{LAT}}\left(t_{\mathrm{j}}\right)\right\rangle$ are the LAT synthetic light curve and its time-average value respectively (with time $t_{j}$ measured in the comoving frame of the blob). The power-law index $\sigma_{\gamma}$, which is theoretically motivated is assumed to be constant in time (for details, see [16]).

4. We numerically compute the broadband SEDs using as an input the generated time series $x(t)$. After transforming the photon spectra to the observer's frame, we compute light curves in X-rays, in $\gamma$-rays, and at OIR wavelengths with a temporal resolution of 1 day. The flux of the disk and BLR components is added to the jet non-thermal emission, whenever relevant.

\footnotetext{
${ }^{3}$ For the $l_{\mathrm{e}}+l_{\mathrm{ext}}$ simulation, we combine variations motivated separately from the same synthetic LAT light curve using $\sigma_{\gamma}=1$.
} 
Table 1: Parameter values of the steady-state models for PKS 2155-304 and 3C 273.

\begin{tabular}{|c|c|c|c|c|c|c|c|c|c|c|c|}
\hline Blazar & & & & & & Parameters & & & & & \\
\hline & $R(\mathrm{~cm})$ & $B(\mathrm{G})$ & $\gamma_{\min }$ & $\gamma_{\max }$ & $p$ & $l_{\mathrm{e}}$ & $l_{\mathrm{ext}}$ & $T_{\text {ext }}(\mathrm{K})$ & $\delta$ & $\Gamma$ & $\theta_{\text {obs }}(\operatorname{deg})$ \\
\hline PKS 2155-304 & $5 \times 10^{16}$ & 0.05 & $4 \times 10^{3}$ & $5 \times 10^{5}$ & 3 & $1.8 \times 10^{-5}$ & $\mathrm{n} / \mathrm{a}$ & $\mathrm{n} / \mathrm{a}$ & 30 & 30 & 1.9 \\
\hline 3C 273 & $6.3 \times 10^{15}$ & 22 & 1 & $3 \times 10^{3}$ & 2.15 & $1.2 \times 10^{-2}$ & $3.3 \times 10^{-2}$ & $3.1 \times 10^{5}$ & 10 & 35 & 4.0 \\
\hline
\end{tabular}

5. We perform timing analysis on the daily-binned model light curves. We compute the coefficient of variation $(\mathrm{CV})$ of simulated light curves as a function of observing frequency. We calculate the discrete correlation function [DCF, 7] for three pairs of light curves ( $J$-band vs. $2-10 \mathrm{keV}$ X-rays, $J$-band vs. $0.1-300 \mathrm{GeV} \gamma$-rays, and $2-10 \mathrm{keV}$ vs. $0.1-300 \mathrm{GeV}$ $\gamma$-rays), and construct colour-magnitude diagrams ( $B-J$ vs. $J$-band).

6. We make a qualitative comparison of our findings with various measures of variability from observations.

\section{Results}

The parameters of the steady-state models used as initial conditions for our time-dependent calculations are summarized in Table 1. In addition to the single-parameter variations, we investigate the coupling of two parameters with an introduced time lag as an additional free parameter. To do so, we perform a simulation of $3 \mathrm{C} 273$ by varying $l_{\mathrm{e}}$ and $l_{\mathrm{ext}}$ assuming a time lag of 100 days in their variations. This lag is a lower limit to the light-crossing time of the BLR region [9]. The results of our time-dependent calculations are presented in Figs. 2-6. A qualitative comparison of our findings with various measures of variability from observations is presented in Table 2.

Variations in $B$ produce significant spectral changes in the SEDs of both sources, as illustrated in Fig. 2, due to the competing cooling processes of radiating electrons (i.e. synchrotron vs. synchrotron self-Compton in PKS 2155-304 and synchrotron vs. external Compton scattering (ECS) in 3C 273). In all other simulations, spectral changes are less important.

The first important test of our simulations is the direct comparison of the $\gamma$-ray flux PDFs as obtained from the theoretical and real Fermi-LAT light curves (see Fig. 3). For PKS 2155-304, the best agreement between simulated and observed PDFs is found for a variable $\delta$. For 3C 273, the best agreement is found for the $l_{\mathrm{e}}$ simulation. The agreement is worse when changes in $l_{\mathrm{e}}$ are coupled to changes in $l_{\text {ext }}$. The inability of the $B$-varying simulations to capture the shape of the observed $\gamma$-ray PDFs stems from the assumption of a constant $\sigma_{\gamma}$ in eq. (1). In the $B$-varying simulations of both sources (and to a lesser extent in the $l_{\mathrm{e}}$-varying simulation of PKS 2155-304), the relative efficiency of synchrotron and Compton cooling processes changes throughout the course of the simulation. As a result, the dependence of the $\gamma$-ray flux on the model parameters changes in time and our assumption breaks down.

One way to quantify the intensity of the variability across the spectrum is to use CVs ${ }^{4}$. The actual $\mathrm{CV}$ values strongly depend on the number of observations $N$ that can differ between

${ }^{4}$ This is defined as $C V=\sqrt{\sigma^{2}} /\langle f\rangle$. Here, $\langle f\rangle$ is the mean and $\sigma^{2}=\sum_{\mathrm{i}=1}^{N}\left(f_{\mathrm{i}}-\langle f\rangle\right)^{2} /(N-1)$ is the variance of the light curve consisting of $N$ data points with fluxes $f_{\mathrm{i}}$ 

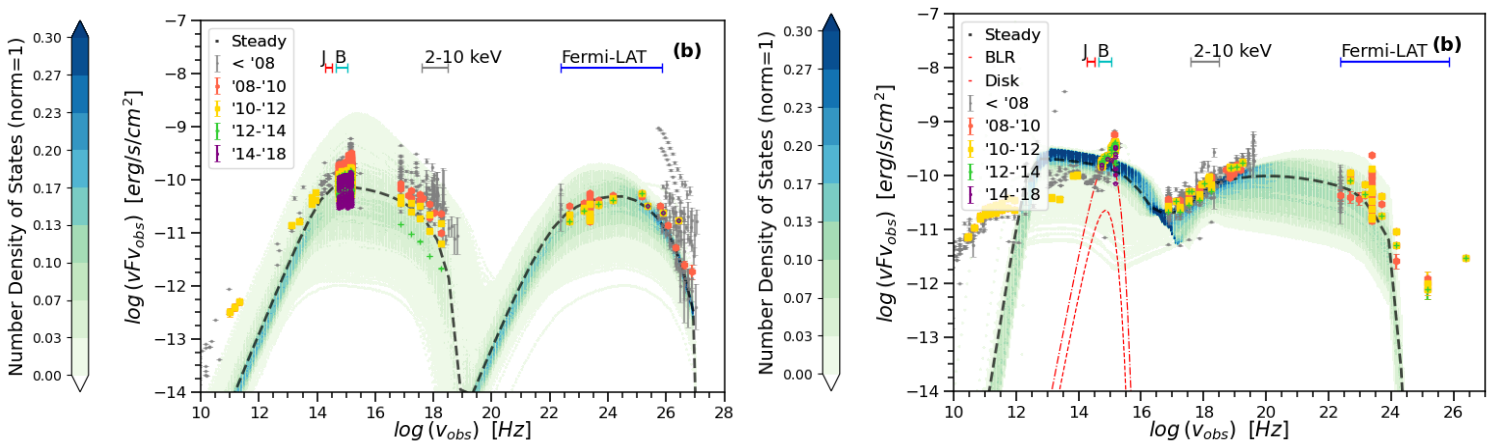

Figure 2: Density map of flux states at different frequencies obtained from our long-term simulations of PKS 2155-304 (left) and 3C 273 (right) with variable $B$. Darker colours suggest that the source spends a larger fraction of simulation time in a particular flux state. The observed SED is compiled using publicly available data (symbols) from the SSDC. Observations prior to 2008 that do not coincide with the 10-yr period of our timing analysis are shown in grey for comparison. The steady-state model is overplotted (dashed black line). Animations of the simulations can be found at YouTube 留e.

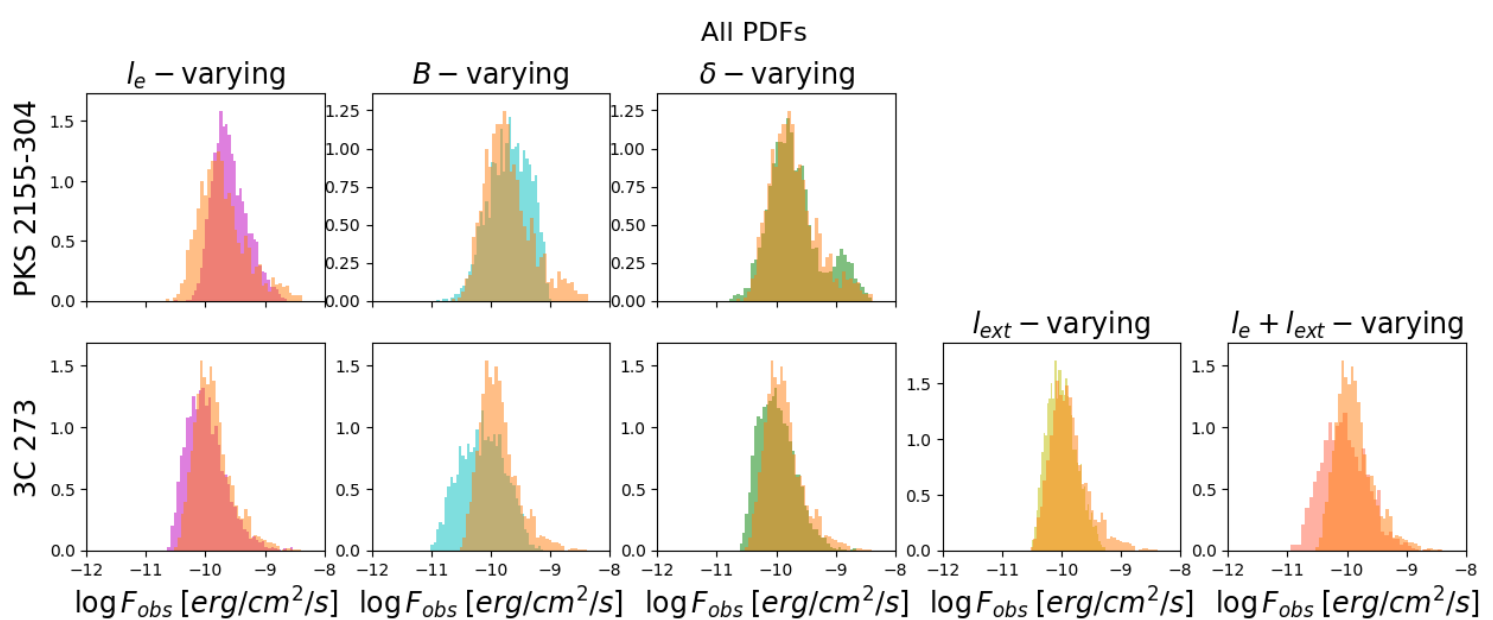

Figure 3: PDFs of observed and simulated $\gamma$-ray fluxes in the $0.1-300 \mathrm{GeV}$ energy range for PKS 2155-304 and 3C 273. The observed PDFs (orange) are created using the $10 \mathrm{yr}$ daily-binned Fermi-LAT light curves. By definition, all histograms are normalized so that the enclosed areas are equal to unity.

observations performed at different energy bands ${ }^{5}$. The most commonly used fractional variability (FV), which corrects for the error bars of the measurements, can be significantly less than the CV value. As shown in Fig. 4, none of the simulations can account for the MW long-term variability of the sources under study. For example, the $\delta$-varying simulations overestimate the variability in OIR and X-rays.

To investigate the correlation and possible time lags between the simulated fluxes in OIR, $\mathrm{X}$-rays, and $\gamma$-rays, we use DCFs [7]. When computing the DCFs for the simulations with varying $l_{\text {ext }}$, we consider both the variable non-thermal jet emission in the $J$-band, but also the time-varying external radiation field. Our results are presented in Fig. 5. All simulations of PKS 2155-304 predict

\footnotetext{
${ }^{5}$ This is especially true if the long-term variability is not a white noise, see Ref. [19].
} 

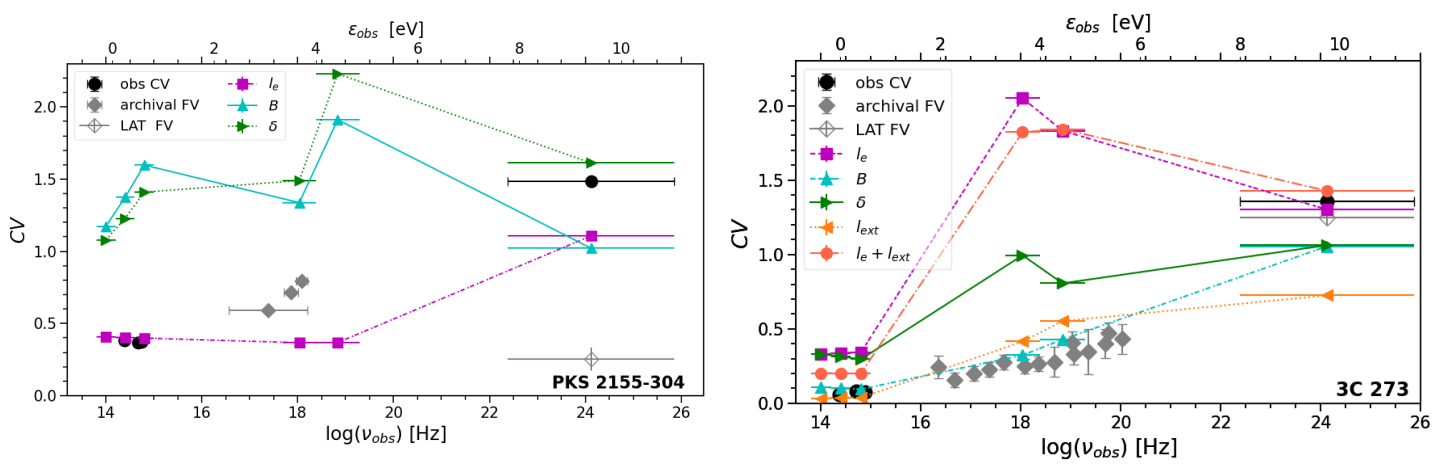

Figure 4: CV as a function of frequency (coloured symbols) computed from our simulations for PKS 2155304 (left panel) and 3C 273 (right panel). Black circles show the CVs in OIR and $\gamma$-ray energies computed from the observed SMARTS and Fermi-LAT light curves. The FV of the LAT light curve is also plotted for comparison (open diamond). FVs from archival observations in ultraviolet and X-rays (grey diamonds) are adopted from [6] and [21] for PKS 2155-304 and 3C 273, respectively.

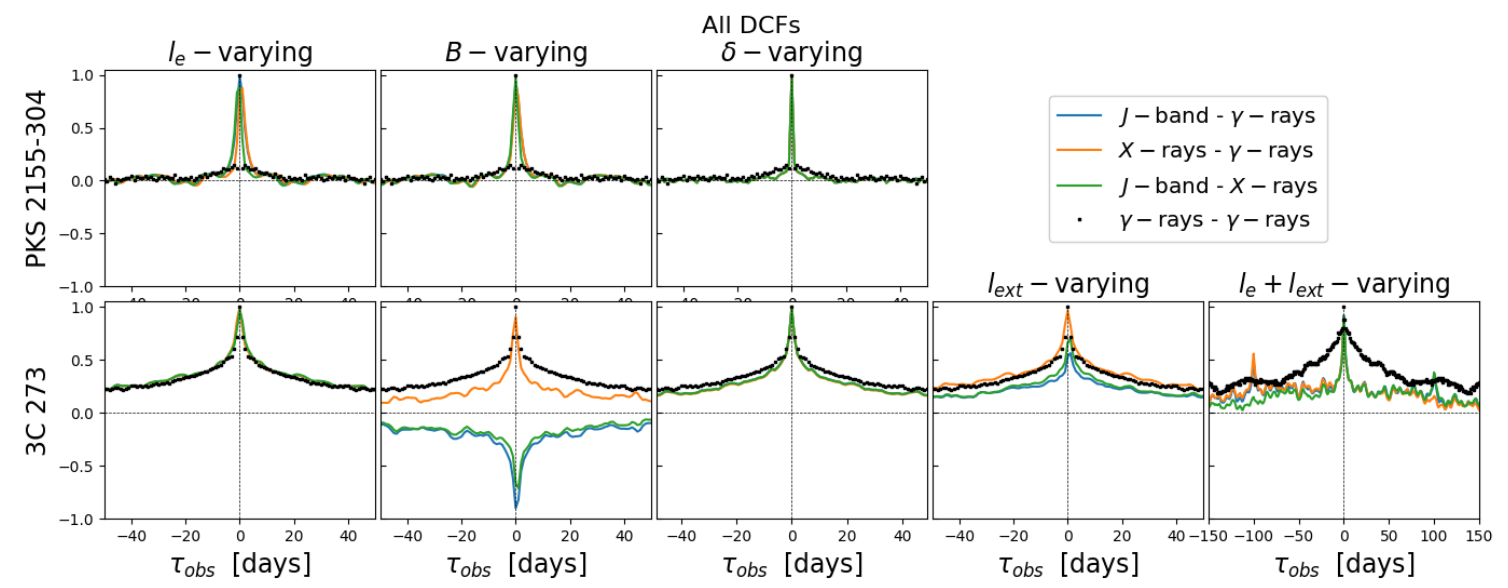

Figure 5: DCFs of the daily binned simulated light curves in the $J$-band, $2-10 \mathrm{keV} X$-rays and $0.1-300 \mathrm{GeV}$ $\gamma$-rays for PKS 2155-304 and 3C 273. A positive time lag, $\tau_{\mathrm{obs}}$, between bands $a$ and $b$ means that the light curve in band $b$ lags behind the light curve in band $a$ by that amount of time. The DCF curves for each source are structured similarly to the auto-correlation function of the input Fermi-LAT light curve (shown in black symbols).

strong positive correlation at zero time lag. These results are not supported by the data, since no correlation between the observed long-term $J$-band and GeV $\gamma$-ray light curves of PKS 2155-304 on timescales $>1$ day was found $[4,10]$. The simulations of 3C 273 show a richer behaviour in the correlations found between the fluxes at different energy bands. For instance, the $B$-varying simulation yields an anti-correlation between the OIR and $\gamma$-ray emission from the jet. A decrease in the magnetic field strength would reduce the synchrotron power and the OIR flux, while channeling more power to the ECS process and increasing the X-ray and $\gamma$-ray fluxes. However, such anticorrelation has not been observed. Instead, a weak correlation at zero time lag was reported for 3C 273 by Bonning et al. [4] using 2 years of SMARTS and Fermi-LAT data [see also 21]. The weak peak at zero time lag was also recovered by a more recent DCF analysis performed on 10 years 

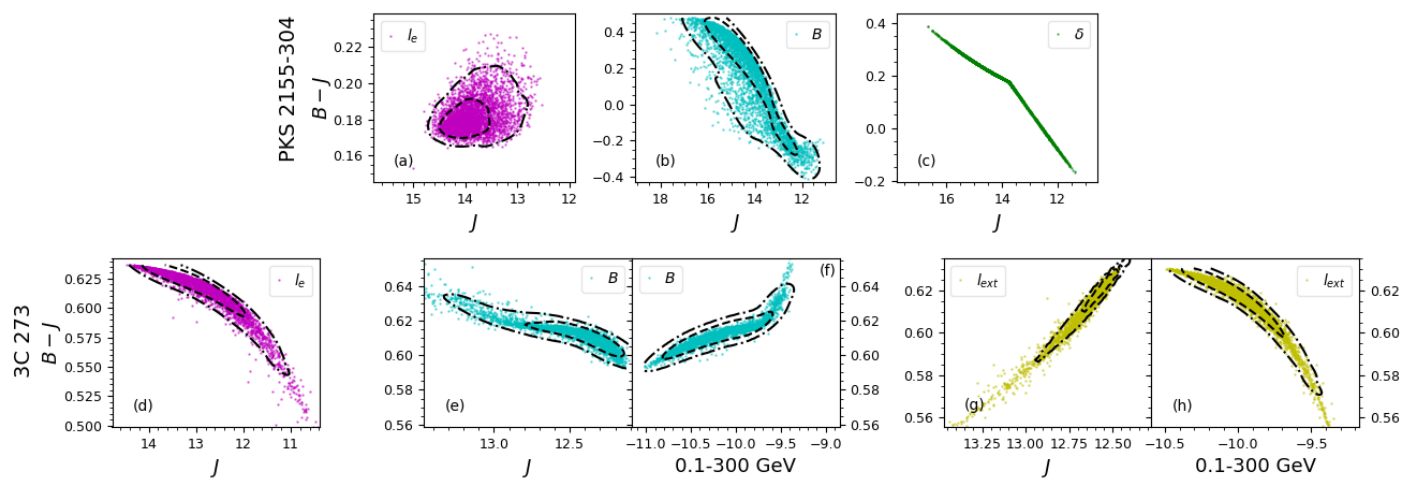

Figure 6: Model-predicted $B-J$ colour plotted against the $J$-band magnitude for PKS 2155-304 and 3C 273. For two simulations of 3C 273 that produce an anti-correlation between OIR and $\gamma$-ray fluxes, the $B-J$ colour versus the $0.1-300 \mathrm{GeV} \gamma$-ray flux is also plotted on the right of the respective colour-magnitude diagram. All other colour- $\gamma$-ray flux diagrams show similar trends as the $B-J$ versus $J$ plots and are not shown here. Dashed (dotted-dashed) lines define the $1 \sigma(2 \sigma)$ standard deviation assuming a Gaussian kernel for the number density of states. For the $l_{\mathrm{e}}+l_{\mathrm{ext}}$ simulation of $3 \mathrm{C} 273$ the $B-J$ versus $J$ plots are similar to the $l_{\mathrm{e}}$ simulation and are therefore not plotted. Note the different axis scales used in different panels.

of SMARTS and Fermi-LAT data [23, 24]. This analysis revealed another peak at lag $~ 50$ days, which cannot be reproduced by any of our simulations with a single-varying parameter. We have shown with our "simplified" two-parameter simulation that a non-zero time lag can be reproduced by the model, when it is induced as an offset in the time series of the varying parameters (see spike at $-100 \mathrm{~d}$, in bottom right plot in Fig. 5). In general, time delays of the order of tens of days are expected due to the physical extension of the BLR.

Table 2: Qualitative comparison of simulations with observations using various diagnostics.

\begin{tabular}{|c|c|c|c|c|c|c|c|c|}
\hline & \multirow{2}{*}{$\begin{array}{c}\gamma \text {-ray PDFs } \\
\text { (1) }\end{array}$} & \multicolumn{3}{|c|}{$\mathrm{FV} / \mathrm{CV}$} & \multirow{2}{*}{$\begin{array}{c}\text { DCF } \\
\text { (J-band vs. } \gamma \text {-rays }) \\
(3)\end{array}$} & \multirow{2}{*}{$\begin{array}{c}B-J \text { vs. } J \text {-band } \\
\text { (4) }\end{array}$} & \multirow{2}{*}{$\begin{array}{c}B-J \text { vs. } \gamma \text {-rays } \\
\text { (5) }\end{array}$} \\
\hline & & & OIR & $\begin{array}{c}\text { X-rays } \\
\text { (2) }\end{array}$ & $\gamma$-rays & & & \\
\hline \multirow{3}{*}{ PKS 2155-304 } & $l_{\mathrm{e}}$ & $?$ & $\checkmark$ & $\searrow$ & $\searrow$ & $x$ & $\checkmark$ & $\checkmark$ \\
\hline & $B$ & $?$ & $\nearrow$ & $\nearrow$ & $\searrow$ & $x$ & $x$ & $x$ \\
\hline & $\delta$ & $\checkmark$ & $\nearrow$ & $\nearrow$ & $\checkmark$ & $?$ & $x$ & $x$ \\
\hline \multirow{5}{*}{$3 \mathrm{C} 273$} & $l_{\mathrm{e}}$ & $\checkmark$ & $\nearrow$ & $\nearrow$ & $\checkmark$ & $x$ & $x$ & $x$ \\
\hline & $B$ & $x$ & $\checkmark$ & $\checkmark$ & $\searrow$ & $x$ & $?$ & $\checkmark$ \\
\hline & $\delta$ & $\checkmark$ & $\nearrow$ & $\searrow$ & $\searrow$ & $x$ & $x$ & $x$ \\
\hline & $l_{\text {ext }}$ & $\checkmark$ & $\checkmark$ & $\checkmark$ & $\searrow$ & $?$ & $\checkmark$ & $x$ \\
\hline & $l_{e}+l_{\mathrm{ext}}$ & $x$ & $\checkmark$ & $\nearrow$ & $\vec{\checkmark}$ & $?$ & $x$ & $x$ \\
\hline
\end{tabular}

(1) The shape of the probability density function of the $\gamma$-ray light curve (see Figure 3 ).

(2) The shape of the multi-frequency coefficient of variation compared to the observed trends (see Figure 4).

(3) Shape of the DCF curves (see blue lines in Figure 5 of this paper and Figure 4 of [4] and [24]).

(4) The shape of the $B-J$ colour versus $J$ magnitude colour diagram compared with observations (see Figure 6 of this paper and Figures 6 and 7 of [18]).

(5) The shape of the $B-J$ colour versus $\gamma$-ray flux colour diagram compared with observations (see Figure 6 of this paper and Figure 6 of [24]).

$\checkmark(\boldsymbol{X})$ Property of the simulated light curve(s) is similar to (inconsistent with) the relevant property from observations.

? Controversial interpretation of the property under examination in the simulated results.

$\searrow(\nearrow)$ The model under-predicts (over-predicts) the observed CV/FV value. 
Colour changes as a function of brightness in OIR wavelengths is another diagnostic of blazar variability usually discussed in the literature [e.g. 11]. In Figure 6 we present the $B-J$ versus $J$-band magnitude (and versus $0.1-300 \mathrm{GeV}$, wherever this is useful) diagrams for both sources. Colour changes need to be more thoroughly investigated with realistic variable underlying thermal components in future studies [15].

\section{Conclusions}

Changes in a single parameter of the one-zone leptonic model describes only partially the variability properties in all three bands (OIR, X-rays, $\gamma$-rays) on long timescales. The shortcomings of our most promising simulations, e.g. the mismatch of the simulated $\gamma$-ray flux-colour diagram ( $l_{\text {ext }}$ simulation of 3C 273) and inability of describing the observed variability in certain bands (e.g. very large X-ray CVs in $l_{\mathrm{e}}$ simulation of PKS 2155-204) suggest that at least two physical parameters have to vary simultaneously to explain the long-term variability across the electromagnetic spectrum for the one-zone model to be viable. Given that each blazar seems to have a distinct "personality", our study scratches the surface of the blazar variability problem and motivates a wider investigation of the SMARTS blazar sample with the methods presented here.

\section{References}

[1] Aharonian F., et al., 2005, A\&A , 430, 865

[2] Asano K., Hayashida M., 2015, ApJL, 808, L18

[3] Atwood W. B., et al., 2009, APJ, 697, 1071

[4] Bonning E., et al., 2012, ApJ , 756, 13

[5] Böttcher M., 2019, Galaxies, 7, 20

[6] Chevalier J., et al., 2019, MNRAS , 484, 749

[7] Edelson R. A., Krolik J. H., 1988, ApJ , 333, 646

[8] Emmanoulopoulos D., McHardy I. M., Papadakis I. E., 2013, MNRAS , 433, 907

[9] Gravity Collaboration et al., 2018, Nat , 563, 657

[10] H. E. S. S. Collaboration et al., 2014, A\&A , 571, A39

[11] Isler J. C., et al., 2017, ApJ , 844, 107

[12] Krawczynski H., Coppi P. S., Aharonian F., 2002, MNRAS , 336, 721

[13] Liodakis I., et al., 2019, ApJ , 880, 32

[14] Mastichiadis A., Kirk J. G., 1997, A\&A , 320, 19

[15] Otero Santos J., et al., 2020, in Contributions to the XIV.0 Scientific Meeting (virtual) of the Spanish Astronomical Society. p. 69

[16] Polkas M., et al., 2021, arXiv e-prints, p. arXiv:2105.07030

[17] Rajput B., Stalin C. S., Sahayanathan S., 2020, MNRAS , 498, 5128

[18] Safna P. Z., et al., 2020, MNRAS , 498, 3578

[19] Schleicher B., et al., 2019, Galaxies, 7, 62

[20] Schmidt M., 1963, Nat, 197, 1040

[21] Soldi S., et al., 2008, A\&A , 486, 411

[22] Wehrle A. E., et al., 2012, ApJ , 758, 72

[23] Yoshida K., et al., 2020, in American Astronomical Society Meeting Abstracts \#235. p. 405.08

[24] Yoshida K., et al., 2021, in preparation 